\title{
New Technology and Approaches for the Acceleration and Enhancement of Microstructural Characterization using Electron Backscatter Diffraction
}

\author{
Matthew M. Nowell ${ }^{1}$, Stuart I. Wright ${ }^{1}$, Travis Rampton ${ }^{2}$, Ryan J. Stromberg ${ }^{3}$, Sanjit Bhowmich ${ }^{3}$, \\ Masateru Shibata ${ }^{4}$ and Natasha Erdman ${ }^{4}$ \\ 1. EDAX Inc., Draper, UT, USA \\ 2. EDAX Inc., Mahwah, NJ, USA \\ 3. Hysitron Inc., Minneapolis, MN, USA \\ 4. JEOL USA Inc., Peabody, MA, USA
}

Electron Backscatter Diffraction (EBSD) has developed into a well-established microstructural characterization technique that provides quantifiable information on the grain size and shape, grain boundary character, preferred orientation, and local strain state of crystalline materials. Recent developments in pattern detection technology have enabled EBSD data acquisitions speeds to increase to rates greater than 1,400 analysed EBSD pattern per second.

To achieve these speeds, acquisition hardware must be coupled with efficient software algorithms that can rapidly both detect the band positions within the EBSD patterns and use this band position information to accurately determine the crystallographic orientation. This image processing requirement is made more difficult at faster acquisition speeds due to typical reduction of the effective EBSD detector resolution. EBSD mapping results from a Nickel alloy, with a cubic crystal structure, collected at 1400 indexed patterns per second with a 99\% indexing success rate and a Titanium alloy, with a hexagonal crystal structure, collected at 1150 indexed patterns per second with a $97 \%$ indexing success rate are shown in Figure 1. Optimization of both camera acquisition and software image processing settings are required to obtain high speed data with acceptable indexing rates.

Sample preparation and SEM operating conditions also play key roles in obtaining faster EBSD acquisition speeds with acceptable indexing success rates. For successful EBSD pattern indexing, the signal to noise ratio (SNR) in the pattern must be sufficiently high to allow accurate band detection. Poor sample preparation reduces SNR while quality sample preparation increases SNR. SNR is also dependent on the camera amplification settings used to collect the digital image. As the amplification level (gain) is increased, SNR decreases. This can be tolerated up until band detection is no longer reliable. An alternative approach is to increase SEM beam current, which increases the incident backscattered electron signal and reduces the time necessary to obtain a useable pattern. With this approach, less electron gain is required and higher SNR patterns are collected. Modern FEG SEM instruments are able to deliver high beam currents without sacrificing spatial resolution.

While 1400 indexing points per second represents a significant increase in acquisition speed for EBSD mapping data, other approaches are able to provide qualitative and quantitative microstructural information more rapidly. One such approach is the use of an EBSD detector as an electron imaging device [1]. With this approach, multiple regions of interest (ROI) are defined within the EBSD phosphor screen, and each ROI is used as a backscattered electron detector and imaging channel. Multiple ROIs (up to 25 with this implementation) can be imaged simultaneously, and these images can provide orientation, deformation, topographic, and atomic number contrast images of the microstructure. Because the camera can be operated at pixel resolutions less than those required for band detection, 
acquisition speeds greater than 2000 frames per second can be achieved. This approach can also be used to determine the position of grain boundaries, which can in turn be used to reduce the number of measurements needed to represent a given microstructure.

The microstructural information obtained can also be correlated with other characterization information. Figure 2 shows an example of the plastic strain field that develops around a nanoindentation. An in situ nanoindentation using PI 87 SEM PicoIndenter (Hysitron, Inc., Minneapolis, USA) was made near a grain boundary, and the plastic strain field, as visualized by the Local Orientation Spread (LOS) map [2] does not propagate through this boundary. As the deformation response is strongly influenced by the local orientation and grain boundary character, the correlation of nanoindentation location with measured microstructure helps better understand local material response [3]. This correlation can be also extended to other microanalysis characterization techniques. For example, Energy Dispersive Spectroscopy (EDS) collected at a lower voltage to improve spatial resolution can be correlated with the EBSD data collected at a higher voltage.

[1] S. Wright et al, Ultramicroscopy 148 (2015), p. 132.

[2] S. Wright, M. Nowell, and D. Field, Microscopy and Microanalysis 17 (2011), p. 316.

[3] C. Fizanne-Michel et al, Materials Science and Engineering A 613 (2014), p. 159.
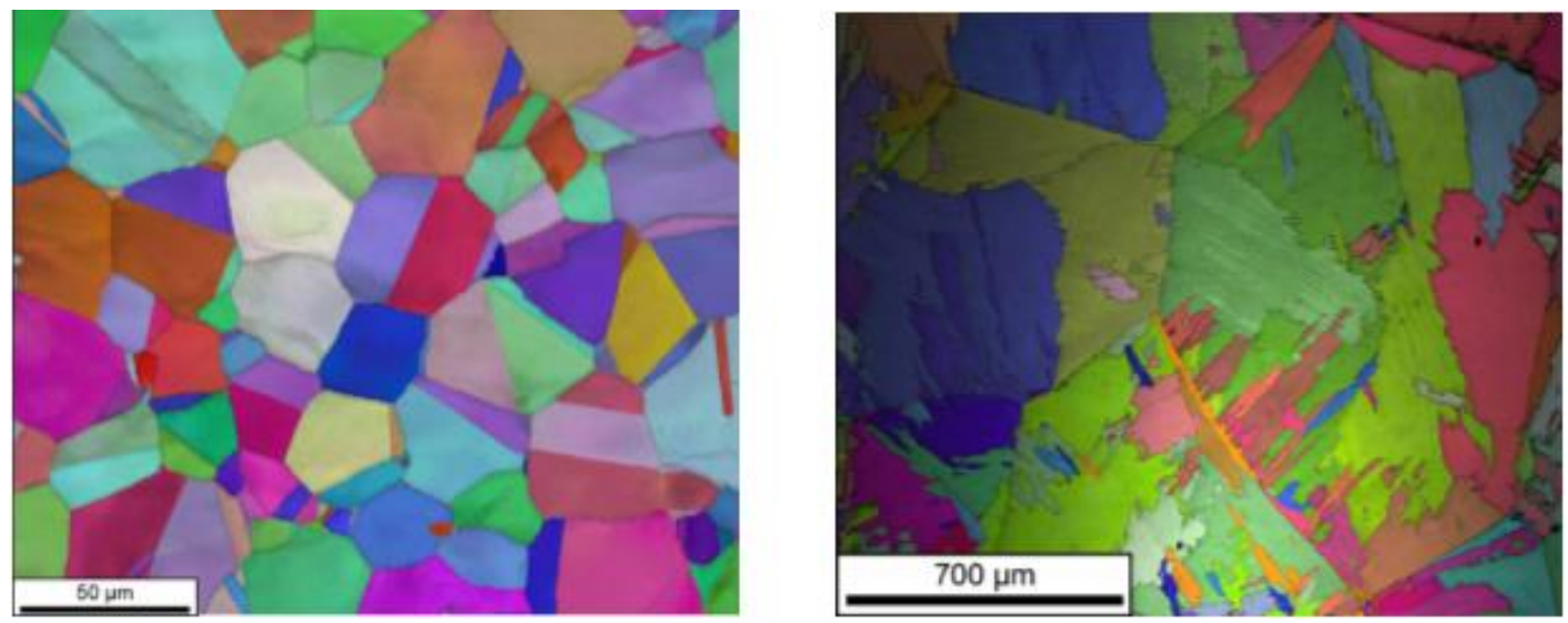

Figure 1. EBSD orientation maps of (left) a nickel alloy collected at 1400 indexed patterns per second with an indexing success rate of $99 \%$ and (right) a titanium alloy collected at 1150 indexed patterns per second with an indexing success rate of $97 \%$.

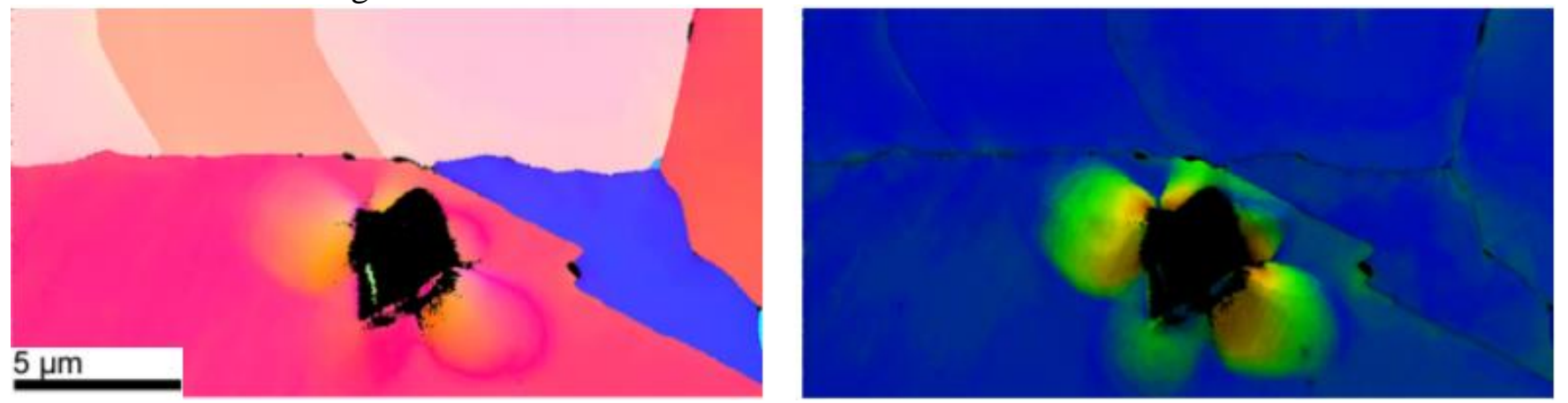

Figure 2. EBSD orientation map (left) and local orientation spread map (right) showing plastic deformation response to nanoindentation near selected grain boundary. 